\title{
Acute neonatal glucocorticoid exposure produces selective and rapid cerebellar neural progenitor cell apoptotic death
}

\author{
KK Noguchi ${ }^{\star, 1}$, KC Walls ${ }^{2}$, DF Wozniak ${ }^{1}$, JW Olney ${ }^{1}$, KA Roth $^{2}$ and NB Farber ${ }^{1}$
}

\begin{abstract}
There has been a growing controversy regarding the continued use of glucocorticoid therapy to treat respiratory dysfunction associated with prematurity, as mounting clinical evidence has shown neonatal exposure produces permanent neuromotor and cognitive deficits. Here we report that, during a selective neonatal window of vulnerability, a single glucocorticoid injection in the mouse produces rapid and selective apoptotic cell death of the proliferating neural progenitor cells in the cerebellar external granule layer and permanent reductions in neuronal cell counts of their progeny, the cerebellar internal granule layer neurons. Our estimates suggest that this mouse window of vulnerability would correspond in the human to a period extending from approximately 20 weeks gestation to 6.5 weeks after birth. This death pathway is critically regulated by the proapoptotic $\mathrm{Bcl}-2$ family member Puma and is independent of p53 expression. These rodent data indicate that there exists a previously unknown window of vulnerability during which a single glucocorticoid exposure at clinically relevant doses can produce neural progenitor cell apoptosis and permanent cerebellar pathology that may be responsible for some of the iatrogenically induced neurodevelopmental abnormalities seen in children exposed to this drug. This vulnerability may be related to the physiological role of glucocorticoids in regulating programmed cell death in the mammalian cerebellum.
\end{abstract}

Cell Death and Differentiation (2008) 15, 1582-1592; doi:10.1038/cdd.2008.97; published online 4 July 2008

The use of perinatal glucocorticoid (GC) therapy for the treatment of respiratory dysfunction associated with prematurity dramatically increased during the 1990s after the National Institutes of Health submitted a consensus statement recommending a single course of antenatal corticosteroids for woman at risk for premature birth. ${ }^{1-3}$ Around this same time, numerous clinicians adopted the use of postnatal and/or more chronic courses of prenatal treatment that were not recommended in the report. ${ }^{2,4}$ Recently, perinatal GC therapy has been mired in controversy due to increasing concerns that it may produce neurodevelopmental abnormalities. ${ }^{5,6}$ Much of this concern derives from several placebo-controlled doubleblind clinical studies which have shown that neonatal GC therapy produces permanent neuromotor and cognitive deficits. ${ }^{7,8}$ Despite rising concerns and no clear mechanism for these impairments, GC therapy has become the standard treatment for women at risk for premature delivery and continues to be used to treat prematurely born infants. 9,10

Previous research in this laboratory has found that several different types of drugs, although relatively innocuous in the adult brain, can produce apoptotic cell death in the developing nervous system. ${ }^{11-13}$ Therefore, the ability of GCs to produce apoptotic cell death was studied in the immature mouse brain to address concerns about the effects of these hormones on neurodevelopment. The findings reveal that, in the mouse, a single GC injection during a developmental age that corresponds to the human perinatal period produces a previously unknown neuropathology involving the rapid and selective apoptotic cell death of the proliferating neural progenitor cells (NPCs) in the cerebellar external granule (germinal) layer (EGL) and permanent reductions in selective cerebellar neuronal populations. This GC-induced NPC death was dependent on the Puma-regulated activation of the intrinsic apoptotic death pathway and is independent of p53 expression.

\section{Results}

GC exposure induces activated caspase-3 immunoreactivity. Caspase- 3 is the major caspase effector in the nervous system and the immunohistochemical detection of cleaved (i.e. activated) caspase-3 (C3A) immunoreactivity has been shown to be a sensitive indicator of apoptosis. ${ }^{14}$ Therefore, to examine the brain for apoptotic cell death, postnatal day (PND) seven C57BL/6 mouse pups were injected with $3.0 \mathrm{mg} / \mathrm{kg}$ of the synthetic GC dexamethasone (DEX) or saline and perfused at various postinjection time points to screen the entire brain for C3A immunoreactivity. In mice perfused 4,6 , and $8 \mathrm{~h}$ postinjection, there was a striking increase in C3A-positive

\footnotetext{
${ }^{1}$ Department of Psychiatry, Washington University School of Medicine, 660 South Euclid Avenue, St Louis, MO 63110-1093, USA and ${ }^{2}$ Department of Pathology, University of Alabama at Birmingham, 1530 Third Avenue, South, SC 930, Birmingham, AL 35294-0017, USA

${ }^{*}$ Corresponding author: KK Noguchi, Department of Psychiatry, Washington University School of Medicine, Campus Box no. 8134, 660 South Euclid, St. Louis, MO 63110, USA. Tel: 314362 2483; Fax: 314362 2474; E-mail: noguchik@ wustl.edu

Keywords: apoptosis; glucocorticoids; corticosterone; cell death; neuropathology

Abbreviations: ANOVA, analysis of variance; AraC, cytosine arabinoside; C3A, activated caspase-3; DEX, dexamethasone; EGL, external granule layer; GC, glucocorticoid; KO, knockout; NPC, neural progenitor cell; PND, postnatal day; WT, wild type

Received 26.3.08; revised 21.5.08; accepted 05.6.08; Edited by JA Cidlowski; published online 04.7.08
} 
cellular profiles in the outer cerebellar EGL of DEX-treated pups when compared to saline controls (Figure 1). At $4 \mathrm{~h}$, C3A immunoreactivity revealed the affected cells to be relatively intact, but in the ensuing $4 \mathrm{~h}$ (i.e. 6 and $8 \mathrm{~h}$ postinjection intervals) they became severely fragmented. There was no increase in $\mathrm{C} 3 \mathrm{~A}$ immunoreactivity at other postinjection time points. In addition, unlike previous research in which neonatal drug exposure induces C3A immunoreactivity throughout the brain, ${ }^{13}$ no qualitative increases could be seen in any other brain region.

To address concerns regarding DEX's limited ability to penetrate the blood-brain barrier ${ }^{15}$ and to verify that other GCs could produce the same NPC apoptosis, the endogenous rodent GC corticosterone, which is highly permeable to the blood-brain barrier, or sesame oil vehicle was administered to PND7 C57BL/6 mouse pups. At $4 \mathrm{~h}$ after exposure to 10 or $5 \mathrm{mg} / \mathrm{kg}$ corticosterone, mice exhibited elevated EGL C3A immunoreactivity that appeared identical to that seen following DEX exposure. Examination of sesame oil controls displayed low levels of C3A immunoreactive NPCs similar to that seen in saline controls. These results confirm an endogenous GC highly permeable to the blood-brain barrier can produce the same pattern of degeneration as that seen with DEX exposure (data not shown).

C3A-positive cells are NPCs. As the EGL is composed of an outer proliferative zone of NPCs and an inner premigratory zone of postmitotic immature neurons, we sought to examine whether the C3A immunoreactive cells were NPCs or premigratory immature neurons through the use of double immunofluorescent detection of C3A and Ki-67 (a proliferative cell marker) $4 \mathrm{~h}$ after a $3.0 \mathrm{mg} / \mathrm{kg}$ DEX injection in PND7 C57BL/6 mice. Dexamethasone exposure led to $\mathrm{C} 3 \mathrm{~A}$ immunoreactive cells well within the Ki-67-defined proliferative zone of the EGL (Figure 2a). This result confirms the C3A-positive cells are NPCs and not immature premigratory neurons.

Confirmation of apoptotic cell death. On the basis of our C3A immunolabeling, we next sought to confirm whether DEX produced NPC death by an apoptotic mechanism by using electron microscopy, the gold standard for the detection of apoptosis. Electron micrographs revealed dramatic increases in NPCs undergoing ultrastructural changes indistinguishable from physiological cell death in animals exposed to $3.0 \mathrm{mg} / \mathrm{kg}$ DEX $4 \mathrm{~h}$ previously (Figure $2 \mathrm{~b}$ and $\mathrm{c}$ ). At the 6 or $8 \mathrm{~h}$ postinjection time points, progenitor cells could be seen in an advanced stage of degeneration and fragmenting into cellular debris. The ultrastructural analysis of saline controls revealed very infrequent NPCs undergoing physiological cell death within the EGL at any postinjection time point. Further analysis with plasticembedded semithin sections stained with methylene blue/ Azure $B$ revealed darkened pyknotic cells located in the superficial portions of the EGL $8 \mathrm{~h}$ after DEX exposure (Figure 2d). These results confirm DEX exposure produces NPC apoptotic cell death within the EGL.

Dose-response curve. Once our initial studies confirmed GCs induce NPC apoptotic death, we sought to further examine this phenomenon by establishing a dose-response curve. C57BL/6 mouse pups were administered a single DEX injection at $3.0,0.3,0.1,0.03,0.01,0.003$, or $0.0 \mathrm{mg} / \mathrm{kg}$ on PND7 and perfused $4 \mathrm{~h}$ later for C3A immunohistochemistry. After semiquantitative degeneration scores were produced for each animal, an $\mathrm{ED}_{50}$ value was defined as the dose that produced a degeneration score halfway between the minimum and maximum values on a calculated four

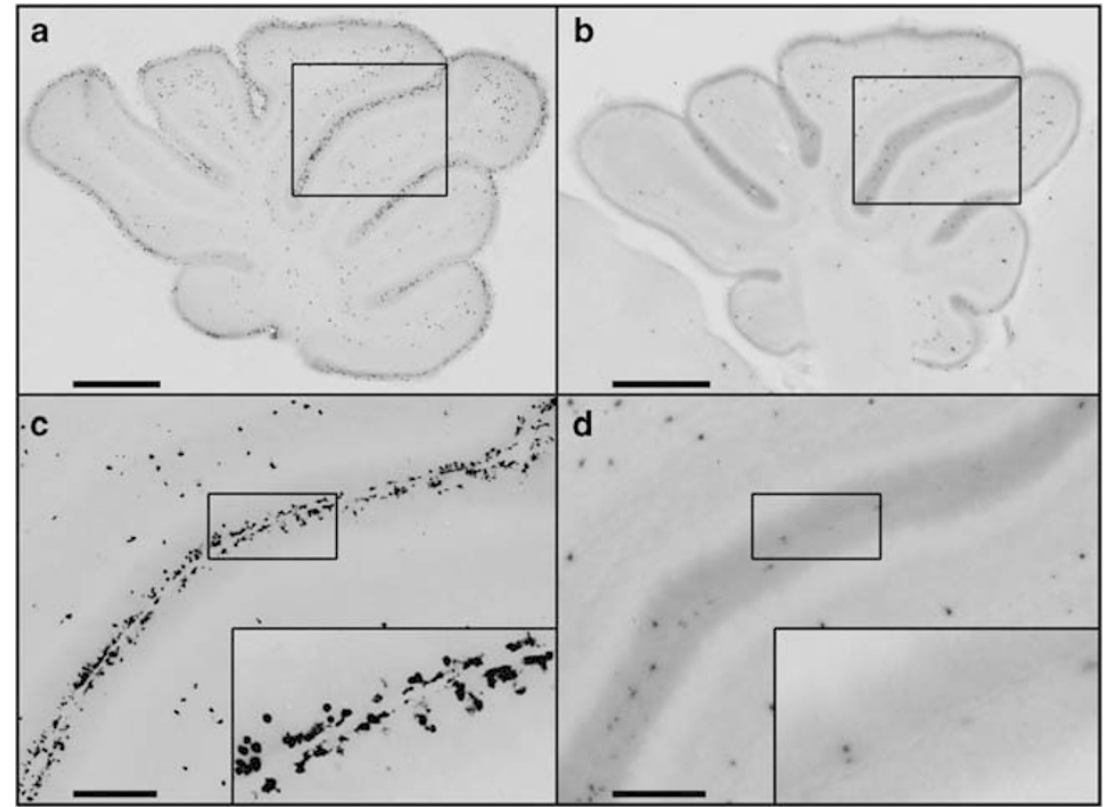

Figure 1 Dexamethasone (DEX) exposure dramatically elevates activated caspase-3 (C3A) immunoreactivity in external granule layer (EGL). Activated caspase-3 immunoreactivity from postnatal day (PND) 7 mouse pups treated with DEX $(\mathbf{a}, \mathbf{c})$ or saline (b, d) $4 \mathrm{~h}$ previously. Boxed regions in (a) and (b) indicate magnified regions depicted in photocomposites (c) and (d), respectively. (c, d) Insets reflect magnified views of boxed regions. Scale bars: (a) and (b) $500 \mu \mathrm{m}$, (c) and (d) $150 \mu \mathrm{m}$ 


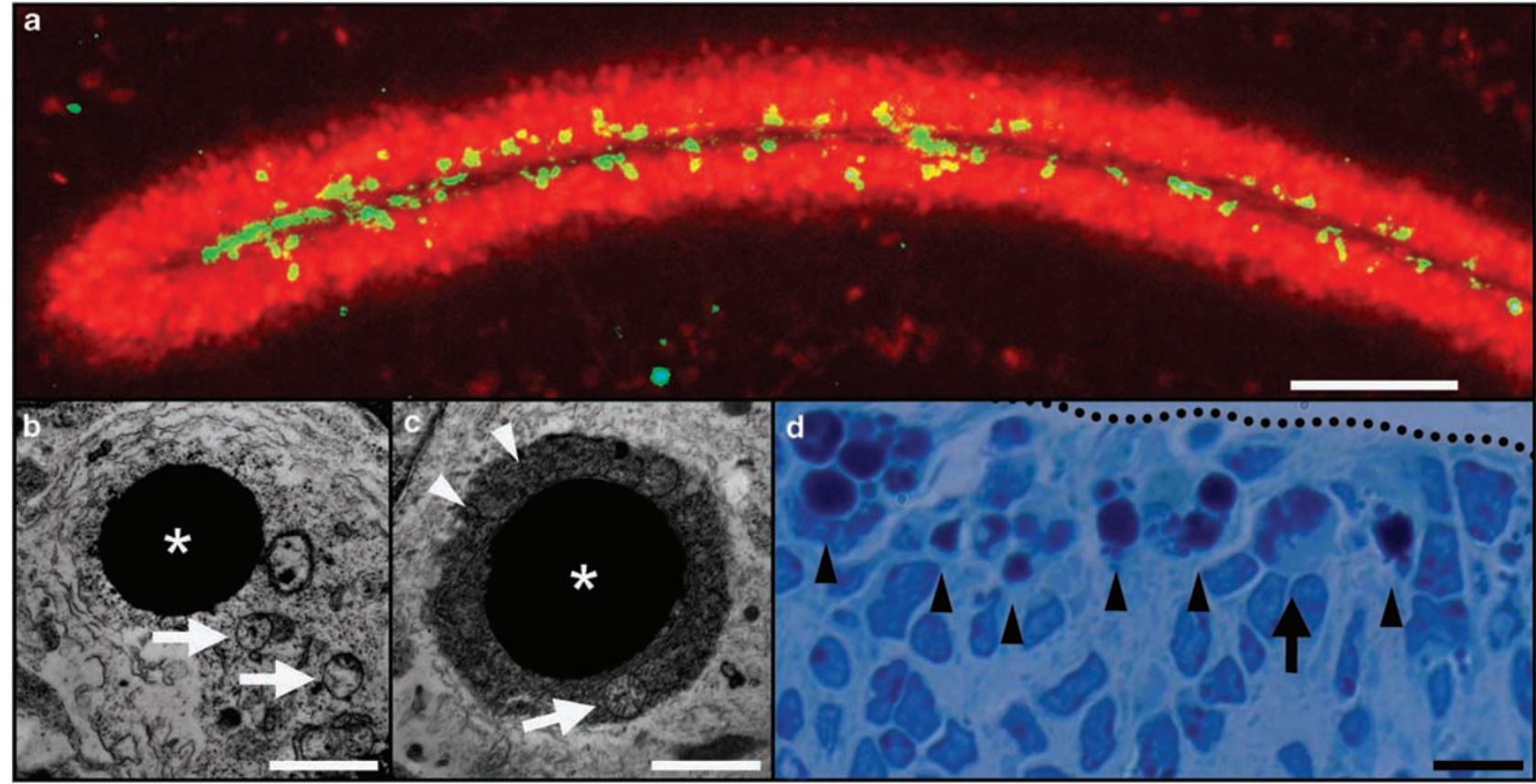

Figure 2 Glucocorticoid (GC) exposure kills neural progenitor cells (NPCs) in the external granule layer (EGL) by apoptosis. (a) Photocomposite of double immunofluorescence $4 \mathrm{~h}$ after $3.0 \mathrm{mg} / \mathrm{kg}$ dexamethasone (DEX) displays activated caspase-3 (C3A)-positive cells (green) within the Ki-67-defined proliferative region (red), indicating degenerating cells are NPCs. (b, c) Electron micrographs of NPCs displaying the classical signs of apoptosis $4 \mathrm{~h}$ after DEX exposure. This includes the formation of chromatin balls $\left({ }^{*}\right)$, cytoplasmic condensation (white arrow heads), and swollen mitochondria (white arrows) undergoing cristolysis and membrane disintegration. (d) Semithin photocomposite $8 \mathrm{~h}$ after DEX exposure. Darkly stained pyknotic NPCs (black arrow heads) can be seen in the superficial portions of the EGL bordering the pial surface (black dotted line) along with a single NPC undergoing mitotic division (black arrow). Scale bars: (a) $100 \mu \mathrm{m}$, (b) and (c) $10 \mu \mathrm{m}$, (d) $20 \mu \mathrm{m}$

parameter logistic equation using Prism software (Graphpad Software Inc., San Diego, CA, USA). The analysis revealed that this drug produced degeneration scores in a dosedependent manner with a calculated $\mathrm{ED}_{50}$ of $0.069 \mathrm{mg} / \mathrm{kg}$ (Figure 3a; 95\% confidence interval: $0.061-0.078 \mathrm{mg} / \mathrm{kg}$ ). Further examination of these data using a one-way analysis of variance (ANOVA) yielded a significant effect of dose $(\mathrm{F}(6,39)=19.3, P<0.0001)$, and subsequent Bonferroni's post hoc comparisons revealed that all doses $0.1 \mathrm{mg} / \mathrm{kg}$ and higher produced significant elevations in degeneration scores when compared to the saline group (Figure 3a).

Developmental window of vulnerability. To assess the developmental window of vulnerability, C57BL/6 mouse pups were administered a single injection of either $3.0 \mathrm{mg} / \mathrm{kg}$ DEX or saline at a selected neonatal age and perfused $4 \mathrm{~h}$ later for semiquantitative evaluation of EGL degeneration. Due to the dramatic changes in the cerebellar morphometry and morphology during the neonatal period, it would be inappropriate to compare degeneration scores between different age groups. As a result, a one-way ANOVA was performed with a planned comparison made only between treatments within each age group. The results revealed a statistically significant difference between groups $(\mathrm{F}(9,61)=70.1, \quad P<0.0001)$. A planned comparison was then performed using a Bonferroni's post hoc analysis. When compared to age matched controls, DEX administration produced no difference in degeneration scores on PND1, a time during which the EGL is a very thin layer of cells covering the cerebellum. Alternatively, DEX produced statistically significant elevations in degeneration scores on PND4 $(P<0.0001)$, PND7 $(P<0.0001)$, and PND10 $(P<0.0001)$. Finally, there was no elevation in degeneration scores at PND14, a time during which much if not all of the EGL has already disappeared (Figure $3 b$ and 4). The results show that there is a window of vulnerability for DEX-induced NPC apoptosis that extends from PND4 through PND10.

Cerebellar GC receptors are localized to the EGL. Previous rodent research using whole region homogenates has found the cerebellum contains the highest levels of $\mathrm{GC}$ receptors in the entire neonatal rodent brain. ${ }^{16}$ Therefore, GC receptor immunohistochemistry was used to determine what subregions of the naive PND7 mouse cerebellum contain this type of receptor. GC receptor immunohistochemistry in the PND7 neonatal mouse revealed that GC receptor immunoreactivity was dramatically elevated throughout the cerebellar EGL (Figure 5a). In addition, lighter GC receptor immunoreactivity was seen in the Purkinje cell layer and white matter regions. To characterize this $\mathrm{GC}$ receptor composition in more detail, GC receptor and glial fibrillary acidic protein (a glial cell marker) immunofluorescence was combined with DAPI staining (a nuclear counterstain) to determine what subcellular regions contain $\mathrm{GC}$ receptors within the EGL. Immunofluorescent examination of the EGL revealed that GC receptor immunoreactivity was primarily contained within the sparse cytoplasmic regions surrounding the DAPI-defined NPC nuclei (Figure 5b). In addition, glial fibrillary acidic 

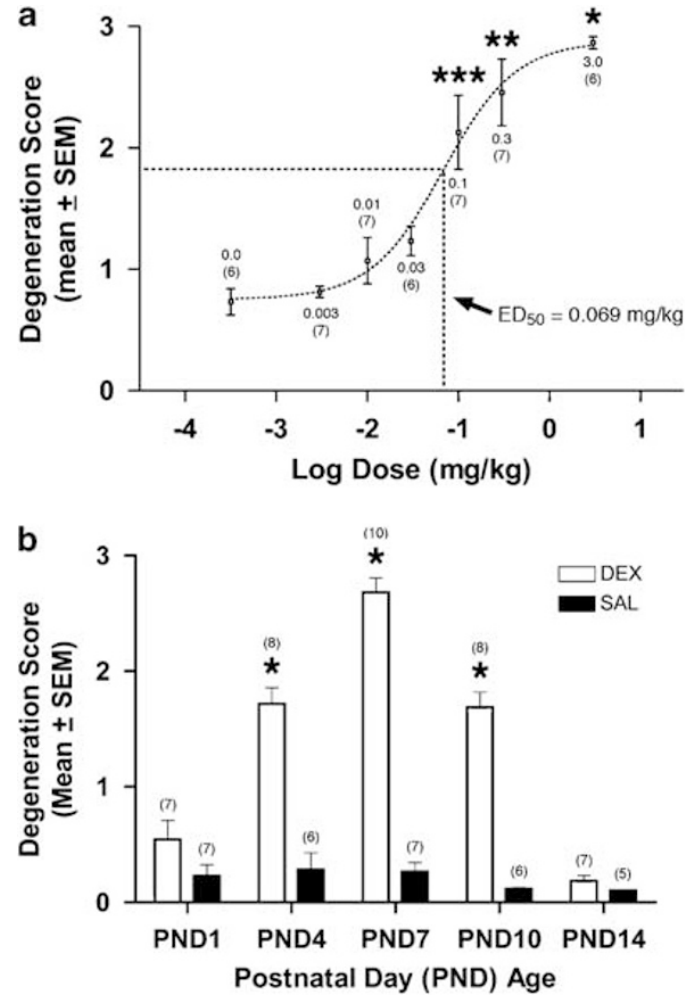

Figure 3 Dose and age effect external granule layer (EGL) degeneration scores. (a) Dose-response curve illustrating semiquantitative degeneration scores in the EGL based on activated caspase-3 (C3A) immunoreactivity following the administration of several different doses of dexamethasone (DEX). The results revealed a calculated $\mathrm{ED}_{50}$ of $0.069 \mathrm{mg} / \mathrm{kg}$ (dotted line) and significant elevations in degeneration scores at doses $0.1 \mathrm{mg} / \mathrm{kg}$ and higher. (b) Drug administration at different neonatal ages revealed that there exists a window of vulnerability extending from postnatal day (PND) 4 to PND10 during which $3.0 \mathrm{mg} / \mathrm{kg}$ DEX significantly increases semiquantitative EGL degeneration scores when compared to an age matched saline group. For all graphs, the numbers above each point represent the dose in $\mathrm{mg} / \mathrm{kg}$ or, if in parenthesis, $n$ per group. Bonferroni's post hoc: significantly elevated compared to saline control group, ${ }^{\star} P<0.0001$, ${ }^{* \star} P<0.01$, ${ }^{* \star \star} P<0.05$

protein immunoreactive Bergmann glia, which provide guidance for newly formed granule cells to migrate to the internal granule cell layer, could be seen projecting to the cerebellar pial surface. Sections containing the hippocampus and paraventricular nucleus were additionally used as positive controls and displayed a pattern of GC immunoreactivity unique to each region. Ultimately, the elevated localization of GC receptors within the NPCs of the EGL suggests that direct stimulation on the progenitors themselves may be mediating their apoptotic cell death.

Long-term consequences of GC exposure. Although we have found $\mathrm{GC}$ exposure produces dramatic increases in EGL NPC apoptosis, it is important to realize this proliferative region naturally disintegrates around 2 weeks of age after cerebellar neurogenesis ends. As a result, any long-term effects of GC exposure should involve a reduction in the number of neurons produced for the internal granule layer due to the premature death of NPCs in the EGL yet not affect other cerebellar regions. Therefore, to asses the long-term effects of GC exposure on the cerebellum, three groups of animals received a single injection on PND7 with saline or DEX (either at 0.3 or $3.0 \mathrm{mg} / \mathrm{kg}$ ) and returned to their dam for care. At 7 weeks of age, each animal was killed and two series of brain sections were labeled with either NeuN, which selectively identifies granule cell neurons within the internal granule layer, or calbindin, which selectively labels Purkinje cells within the Purkinje cell layer were prepared. Stereological cell counts revealed that a single DEX exposure on PND7 significantly reduced the number of NeuN-positive granule cell neurons in the internal granule layer, the sole recipient of neurons produced by the EGL (Figure 6a; $\mathrm{F}(2,20)=30.82, P<0.0001)$. This loss of neurons occurred in a dose-dependent fashion with a single 3.0 or $0.3 \mathrm{mg} / \mathrm{kg}$ DEX injection, reducing the average number of NeuN-positive neurons by 18 or $6 \%$ respectively, when compared to saline exposed littermates. In contrast, the number of calbindin-positive neurons in the cerebellar Purkinje cell layer, a population of cerebellar neurons formed prior to birth by a different proliferative region and whose numbers are not affected by reductions in internal granule layer neurons, ${ }^{17}$ was not altered by exposure to DEX (Figure 6b; $\mathrm{F}(2,20)=0.2837 ; P>0.7$ ).

To obtain preliminary evidence about whether this DEXinduced pathology is associated with persisting behavioral deficits, PND7 C57BL/6 mice were exposed to 3.0, 0.3, or $0.0 \mathrm{mg} / \mathrm{kg}$ DEX and evaluated on a 1-h locomotor activity/ exploration test on PND24. Groups differed significantly in terms of rearing $(F(2,67)=3.83, P<0.05)$, and subsequent comparisons showed that differences were mostly due to the $3.0 \mathrm{mg} / \mathrm{kg}$ DEX group exhibiting significantly fewer rearings compared to saline controls $(P<0.025)$, whereas the $0.3 \mathrm{mg} /$ $\mathrm{kg}$ DEX group did not differ from the control mice (Figure 6c). No significant group differences were found on any other activity variable (Figure $6 d-h$ ).

Intracellular signaling mechanisms. To begin to define the molecular pathways regulating DEX-induced NPC apoptosis, we examined the effects of $3.0 \mathrm{mg} / \mathrm{kg}$ DEX on PND7 mice with targeted gene disruptions in specific apoptosis-associated genes and their wild-type (WT) littermates. Apoptosis can be triggered either through the mitochondrial-dependent intrinsic apoptotic pathway or the cell death receptor-mediated extrinsic apoptotic pathway. ${ }^{18}$ The intrinsic pathway is critically regulated by Bax and Bak that are multi-BH domain, proapoptotic members of the $\mathrm{Bcl}-2$ family. Mice deficient in Bax and Bak exhibit expanded NPC populations in the postnatal brain indicating that programmed cell death of NPCs is normally regulated by the intrinsic apoptotic death pathway. ${ }^{19}$ Bax, Bak dual-deficient NPCs are markedly resistant to a wide variety of death stimuli both in vivo and in vitro. Similar to saline injected WT mice, DEXinjected Bax, Bak dual-deficient PND7 mice showed no evidence of cerebellar EGL apoptosis by histological analysis and $\mathrm{C} 3 \mathrm{~A}$ was undetectable by both immunohistochemical staining of cerebellum (Figure 7a and $\mathrm{c}$ ) and western blot of cerebellar extracts (Figure 8).

Previous studies of DEX-induced apoptosis of lymphocytes indicate that Puma (p53 upregulated mediator of apoptosis), a $\mathrm{BH} 3-$ only proapoptotic member of the Bcl-2 family, is a key upstream regulator of DEX-induced lymphocyte death. ${ }^{20}$ To 


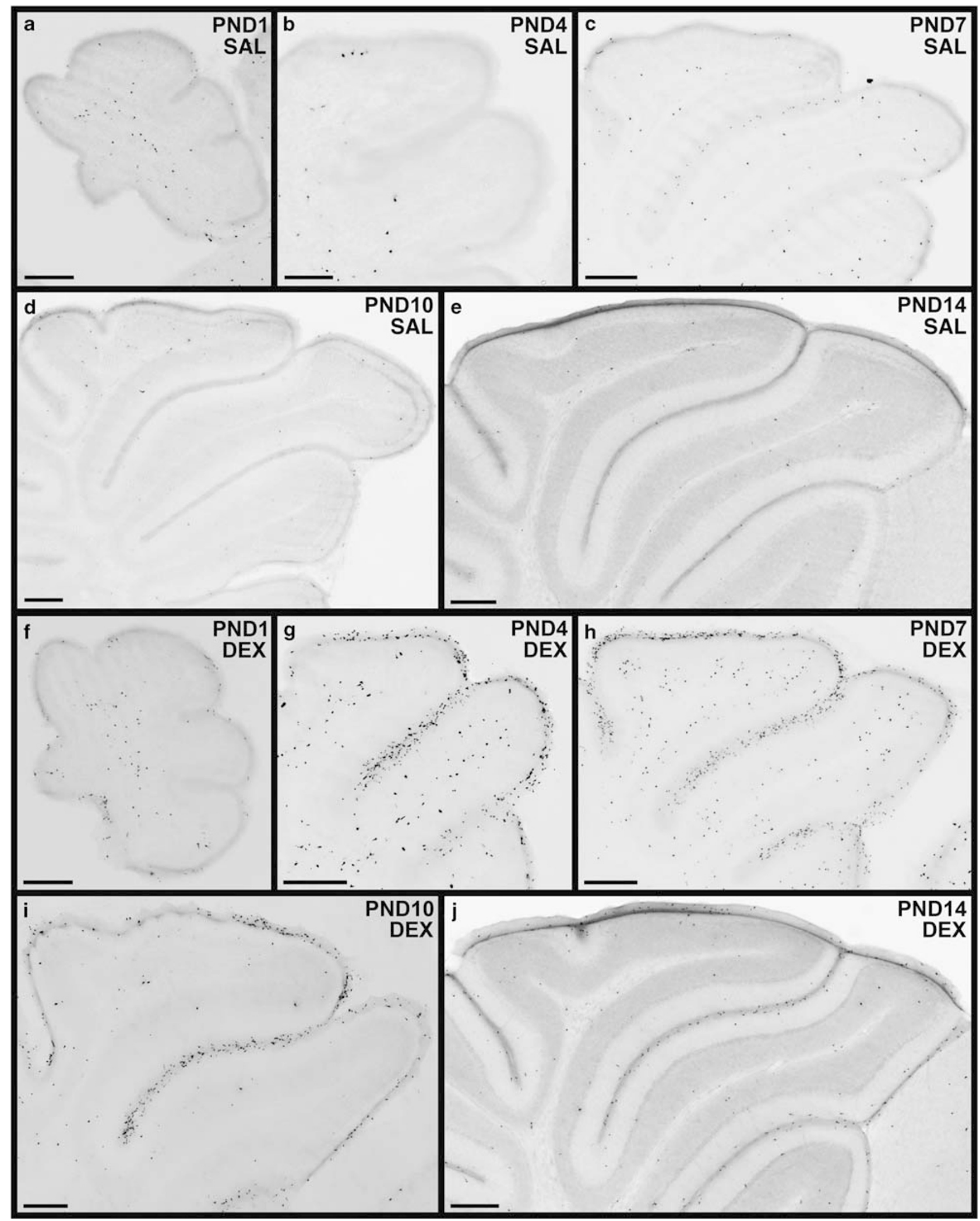

Figure 4 Activated caspase-3 immunoreactivity at several neonatal ages. Photocomposites depicting the activated caspase-3 (C3A) immunoreactivity typically seen $4 \mathrm{~h}$ following either a saline (SAL; a-e) or $3.0 \mathrm{mg} / \mathrm{kg}$ dexamethasone (DEX) injection $(\mathbf{f}-\mathrm{j})$ at different ages. The images indicate DEX exposure produces immunoreactivity comparable to that seen in saline controls on postnatal day (PND) 1 (a, f), increased immunoreactivity on PND4 (b, g), PND7 (c, h), and PND10 (d, i), and no increase in immunoreactivity on PND14 (e, j). Scale bar: $250 \mu \mathrm{m}$ 


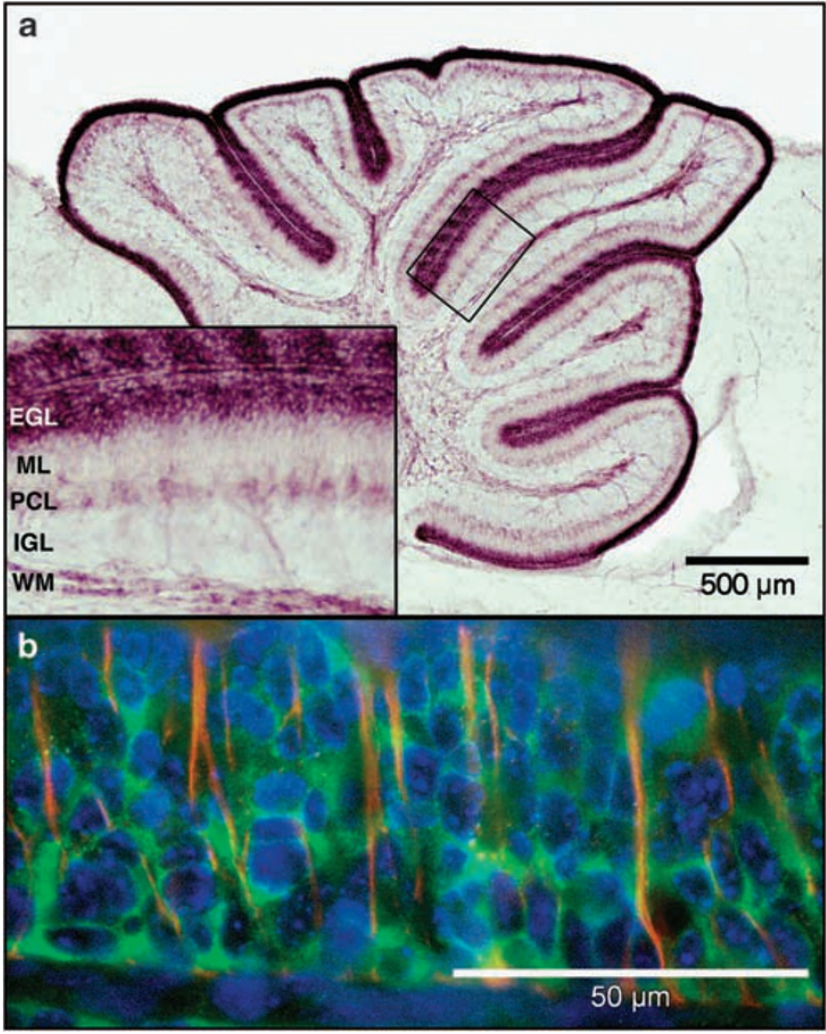

Figure 5 Glucocorticoid (GC) receptor immunoreactivity in the cerebellum. (a) GC receptor immunohistochemistry in the postnatal day (PND) 7 mouse pup shows high levels of immunoreactivity in the external granule layer (EGL), moderate levels in the Purkinje cell layer (PCL), and white matter (WM) regions, and low levels in the molecular layer (ML) and internal granule cell layer (IGL). Inset reflects magnified views of boxed region. (b) Fluorescent immunolabeling reveals GC receptor immunoreactivity (green) in the sparse cytoplasmic regions surrounding the DAPI-labeled (blue) nuclei in addition to glial fibrillary acidic protein (red) immunoreactive Bergmann glia

determine if Puma plays a similar role in DEX-induced apoptosis of NPCs, we compared the effects of DEX on WT and Puma-deficient PND7 mice. Puma-deficient mice, following DEX treatment, showed markedly attenuated caspase-3 activation and apoptosis compared to WT littermates indicating that Puma is an important upstream mediator of DEXinduced apoptosis (Figure 7b, $d$ and 8).

Puma expression is typically regulated by p53 transcriptional activity. However, DEX-induced apoptosis of lymphocytes occurs independently of p53 and Puma expression may be upregulated by other transcription factors. ${ }^{20}$ Thus, we examined the effects of DEX on cerebellar EGL NPCs in p53deficient PND7 mice. Dexamethasone-induced caspase-3 activation (Figure 8) and NPC apoptosis were unaffected by p53 deficiency. These results suggest that Puma acts independently of p53 to regulate DEX-induced NPC apoptosis. To further test this hypothesis, we compared the effects of the genotoxic agent, cytosine arabinoside (AraC), which we have previously shown acts through p53-dependent transcriptional upregulation of Puma expression, with those of DEX in WT and Puma-deficient PND7 mice. ${ }^{21}$ WT cerebellar NPCs show significantly increased C3A (which we have described previously ${ }^{21}$ ) and p53 immunoreactivity following AraC exposure (Figure 9). In comparison, Puma-deficient cerebellar NPCs exposed to AraC show minimal C3A immunoreactivity but markedly increased nuclear p53 immunoreactivity consistent with Puma lying between p53 and caspase-3 in a linear apoptotic death pathway. In contrast to AraC, DEX exposure failed to affect p53 immunoreactivity in either WT or Puma-deficient cerebellar NPCs indicating that p53 is uninvolved in this alternative Puma-dependent apoptotic death pathway (Figure 9).

\section{Discussion}

The NPCs of the EGL produce granule cell neurons for the cerebellar internal granule layer, a homogeneous population of neurons so numerous they represent over half the neurons in the brain. ${ }^{22}$ Here we show that there exists a previously unrecognized window of vulnerability during which a single GC injection produces rapid and selective apoptotic cell death in the NPCs of the EGL. Previous research has established that manipulating an exponentially expanding population of NPCs through apoptosis can affect neurodevelopment by dramatically changing the amount of neurons a proliferative region can produce. ${ }^{23-25}$ As changes in cell size and shape can bias neuronal counts when done with typical crosssectional counting, we used unbiased stereology, a counting methodology not influenced by such changes, to examine the effects of GC exposure on cerebellar neuronal counts for the first time. Our unbiased stereological cell counts show that this same GC exposure produces permanent decreases in the number of NeuN-positive granule cells in the internal granule layer but no changes in the number of calbindin-positive Purkinje cells, an adjacent cerebellar layer whose neurons are produced by a separate proliferative layer.

Clinical implications. Due to the high degree of conservation in cerebellar anatomy, function, and development between the mouse and human, ${ }^{26}$ a similar toxicity may occur following GC exposure in the human perinatal clinical setting. As extrapolating developmental periods from rodents to humans can vary notably dependent on brain region, normalized EGL thickness ratios were used to more accurately estimate an equivalent human window of vulnerability based on our results. Using our mouse developmental window of vulnerability extending from PND4 through PND10, we calculated an equivalent human developmental period would extend from approximately 20 weeks gestation to 6.5 weeks after birth (Figure 10). As the fetus is not considered viable outside the womb prior to 20 weeks gestation, this window of vulnerability, if correct, would include all fetuses receiving the prenatal corticosteroid therapy recommended by the National Institutes of Health for women at risk for premature birth, ${ }^{1,2}$ all prematurely born infants, and extend to the early neonatal period in full-term newborns. In the postnatal clinical setting, DEX dosing regimens typically range from 0.25 to $1.0 \mathrm{mg} / \mathrm{kg}$ and can be given twice daily for up to a month at a tapering dose. ${ }^{7,8}$ Our results show that a single injection of DEX produces $E G L$ degeneration with a calculated $E D_{50}$ of $0.069 \mathrm{mg} / \mathrm{kg}$, leads to statistically significant elevations in 
Longterm Stereological Cell Counts
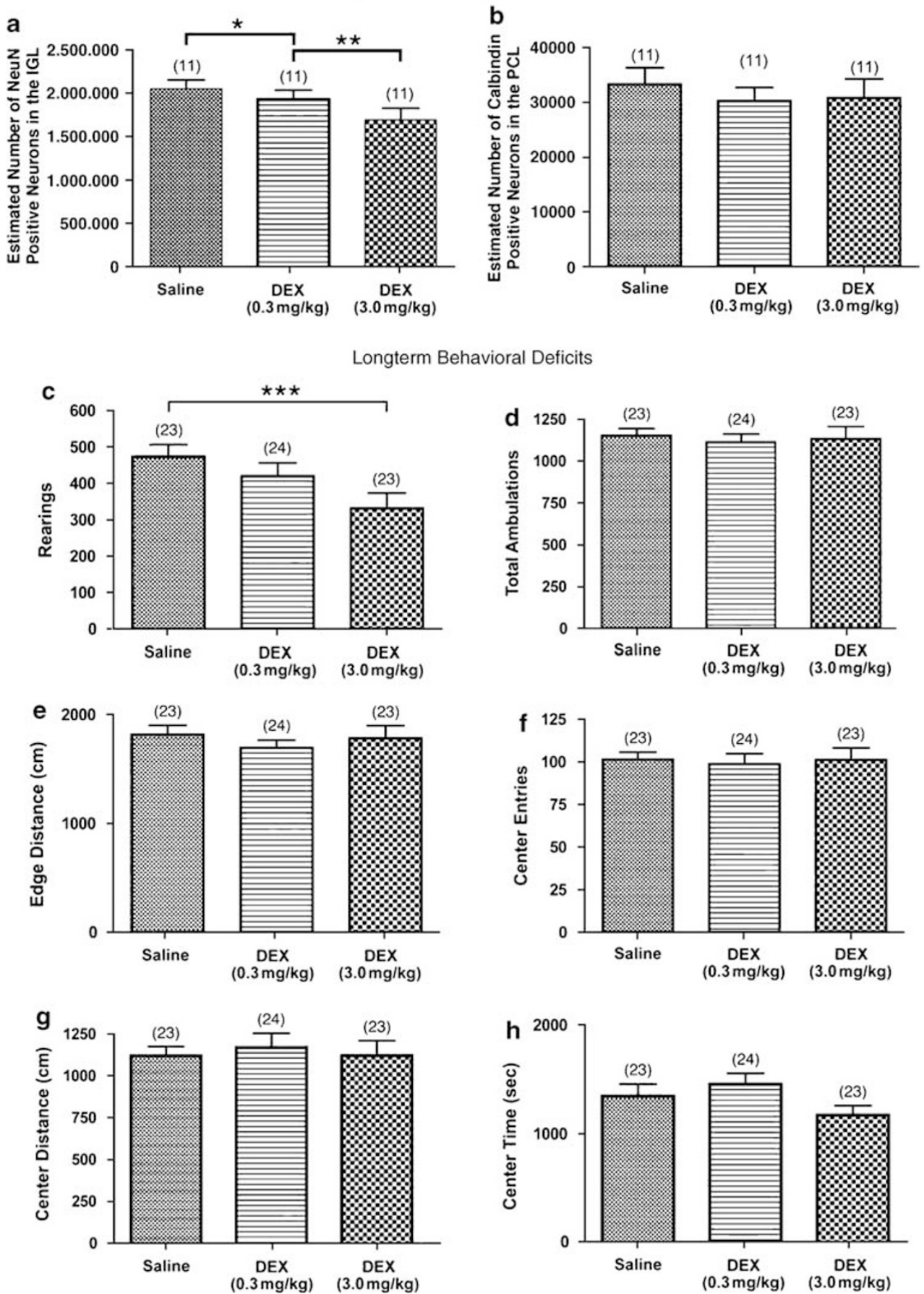

Figure 6 Neonatal dexamethasone (DEX) exposure produces permanent reductions in selective cerebellar neuronal populations and long-term locomotor behavioral deficits. (a) A single injection of 0.3 or $3.0 \mathrm{mg} / \mathrm{kg}$ DEX on postnatal day (PND) 7 produced a significant reduction in the estimated number of NeuN-positive granule cell neurons in the internal granule layer of the cerebellum at 7 weeks of age. (b) An estimation of the number of calbindin-positive Purkinje cell neurons in the Purkinje cell layer of the cerebellum in the same animals revealed no significant differences. Examination of several locomotor behavioral measures on PND24 after neonatal DEX exposure revealed significant deficits in rearings in the $3.0 \mathrm{mg} / \mathrm{kg}$ group (c) but no other significant effects $(\mathbf{d}-\mathrm{h})$. All values are mean \pm S.E.M. with $n$ per group in parenthesis. Bonferroni post hoc ${ }^{\star} P<0.05 ;{ }^{* *} P<0.001 ;{ }^{* \star} P<0.025$

degeneration scores at all doses tested above this $\mathrm{ED}_{50}$, and produced permanent decreases in NeuN-positive granule cells at or above $0.3 \mathrm{mg} / \mathrm{kg}$. According to previously published rodent research, these doses are considered well within the clinically relevant range for neonatology and obstetrics. ${ }^{27}$ Of equal importance, iatrogenically induced cerebellar dysfunction ${ }^{28}$ may explain some of the neuromotor and cognitive deficits described in prematurely born human neonates exposed to chronic GC dosing regimens, ${ }^{7,8}$ as clinical research has firmly established that cerebellar 

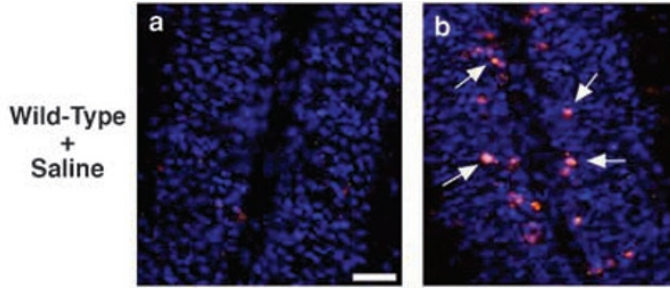

Wild-Type DEX
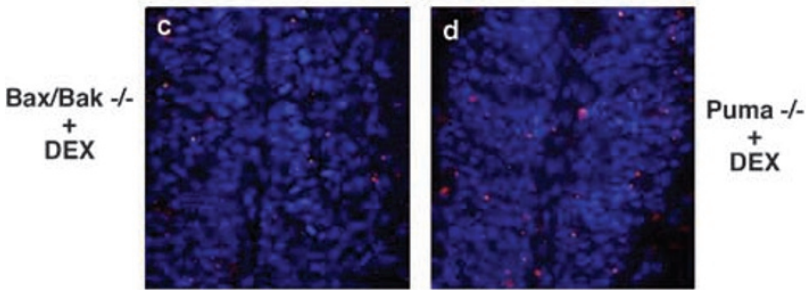

Figure 7 Dexamethasone (DEX)-induced neural progenitor cell (NPC) apoptosis is dependent on Puma and Bax/Bak expression. Postnatal day 7 pups were injected with $3.0 \mathrm{mg} / \mathrm{kg}$ DEX or saline and killed $8 \mathrm{~h}$ later. (a) Activated caspase-3 immunoreactivity was virtually undetectable in the saline-treated wildtype (WT) cerebellum. (b) DEX treatment of WT mice produced numerous cells in the cerebellar external granule layer (EGL) with activated caspase-3 (C3A) immunoreactivity (red; examples indicated by arrows). DEX-induced C3A was completely inhibited in Bax/Bak-/ - mice (c) and greatly attenuated in Puma-/mice (d). Nuclei are labeled blue with bisbenzimide. Scale bar: $20 \mu \mathrm{m}$
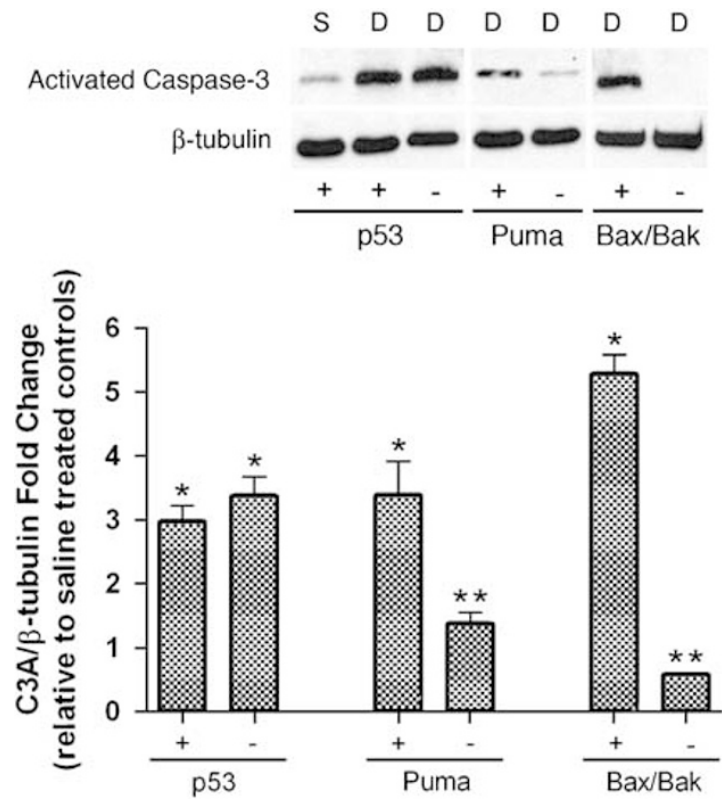

Figure 8 Dexamethasone (DEX)-induced caspase-3-cleavage is dependent on Puma and Bax/Bak expression but not p53. Postnatal day 7 pups were injected with $3.0 \mathrm{mg} / \mathrm{kg}$ DEX (D) or saline (S), killed $8 \mathrm{~h}$ later, and cerebellar lysates prepared. Treatment with DEX significantly increased levels of activated caspase-3 (C3A) in wild type (WT) and p53-1- brains but did not increase C3A levels in Puma- - - or Bax/Bak- $/$ - brains. $\beta$-Tubulin was used as a loading control. C3A levels were significantly reduced in Puma- $/-$ and Bax/Bak $-1-$ mice treated with DEX in comparison to WT DEX-treated mice. Western blot data are represented graphically (mean \pm S.E.M.) as the fold change in ratio of C3A to $\beta$-tubulin relative to salinetreated controls. Results are representative of three littermate pairs (WT and knockout, KO) for each gene disruption (p53, Puma, and Bax/Bak). ${ }^{*} P<0.05$ versus saline control; ${ }^{* \star} P<0.05$ versus wild-type DEX treated pathology can produce both neuromotor and cognitive dysfunction. ${ }^{28}$ Consistent with this hypothesis, we found that neonatal DEX can lead to persistent deficits in rearing on the 1-h locomotor test, a finding often observed in ataxic mice. Although decreased rearing may result from alterations in exploratory behaviors, it probably reflects hindlimb impairments as the groups did not differ on any other activity variable. More extensive behavioral testing is needed to clarify the exact nature of the deficit. As a result, these findings raise new questions about the safety of GCs as rates of premature birth have dramatically increased in recent years ${ }^{29}$ and GC therapy has become the standard treatment for women at risk for premature birth. ${ }^{10}$

Glucocorticoids and cerebellar neurodevelopment. The extremely selective susceptibility of the EGL NPCs to apoptotic cell death following neonatal GC exposure distinguishes it from other types of drug-induced cell death we have found ${ }^{11-13}$ and may suggest this hormone plays a special role in cerebellar development. If true, this may explain the intimate timing and control the rodent body maintains over endogenous GC exposure to the EGL. For instance, when EGL NPCs are expected to undergo extensive expansion and proliferation, they are protected from GC stimulation by low amounts of endogenous corticosterone release $^{16,30}$ and high levels of a GC metabolizing enzyme selectively protecting the EGL NPCs. ${ }^{31,32}$ As EGL neurogenesis nears completion and the NPCs are no longer needed, increased GC release and reductions of this same enzyme conspire to increase stimulation on the high density of GC receptors in the EGL. This information, when combined with our finding that GC exposure produces NPC apoptosis, may suggest that changes in proliferation and the disappearance of the $E G L$ may be due, at least in part, to the increased endogenous GC stimulation and its resulting apoptosis. Ultimately, a regulatory role for GCs may not be surprising because previous research has established that endogenous GCs can regulate apoptosis in other cell populations ${ }^{33}$ or act as a perinatal maturational signal in multiple organ systems. ${ }^{34}$

Intracellular signaling mechanisms. Our studies of DEXinduced NPC apoptosis emphasize the complex and stimulus-specific nature by which NPC apoptosis is triggered. GCs show a variety of cell type-specific pro- and antiapoptotic effects and the molecular pathways that regulate these disparate actions are complex. Interestingly, DEX-induced death of lymphocytes and NPCs appear to follow a similar molecular sequence involving p53-independent, Puma-dependent activation of the Bax, Bak-dependent intrinsic apoptotic death pathway. Although Puma deficiency significantly inhibited caspase-3 activation and NPC apoptosis, this effect was not as pronounced as that produced by Bax, Bak dual deficiency suggesting that still other members of the $\mathrm{BH} 3$-only $\mathrm{Bcl}-2$ subfamily may be involved in triggering GC-induced NPC apoptosis. It was recently reported that DEX-induced lymphocyte apoptosis may involve both Puma and Bim. ${ }^{35}$ We speculate that Bim, or still other members of the BH3-only subfamily such as Noxa, may contribute to DEX-induced NPC apoptosis. 

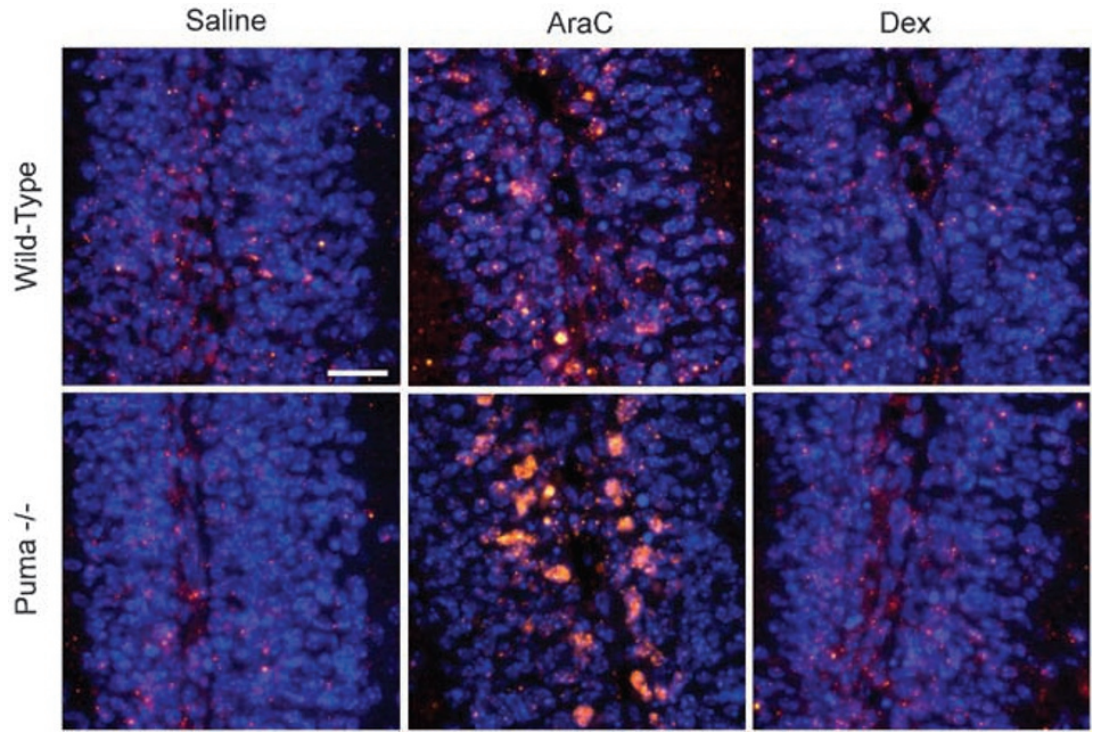

Figure 9 Cytosine arabinoside (AraC)-induced neural progenitor cell (NPC) death increases p53 immunoreactivity unlike dexamethasone (DEX)-induced NPC death Wild-type and Puma-deficient mice were injected with DEX $(3.0 \mathrm{mg} / \mathrm{kg})$ or AraC $(25 \mathrm{mg} / \mathrm{kg})$ and killed $6-8 \mathrm{~h}$ later. Wild-type and Puma-deficient cerebellar NPCs exposed to AraC exhibited increased p53 immunoreactivity (red) in comparison to saline-treated brains. DEX had no effect on p53 immunoreactivity in either WT or Puma-deficient NPCs. Nuclei are labeled blue with bisbenzimide. Scale bar: $20 \mu \mathrm{m}$

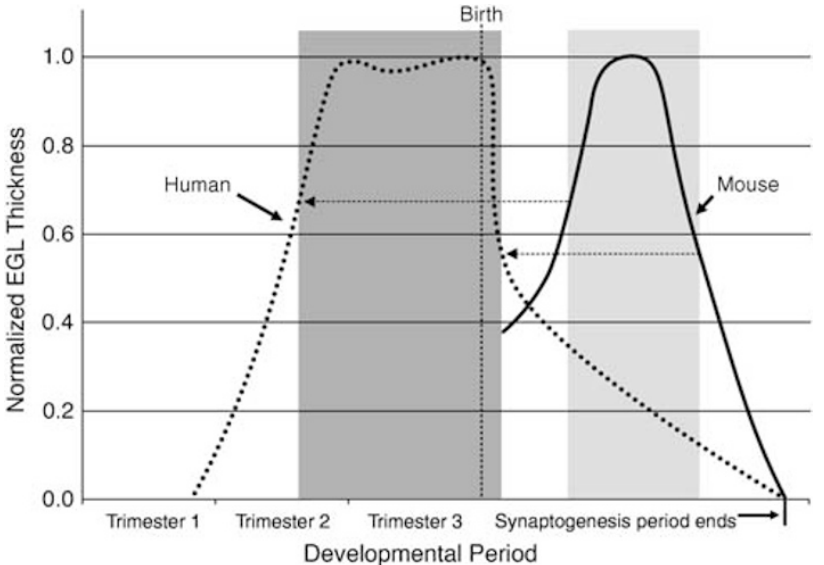

Figure 10 Estimation of an equivalent developmental window of vulnerability in the human. On the basis of our mouse window of vulnerability for DEX-induced NPC apoptosis that extends from postnatal day (PND) 4 through PND10 (light shading), a corresponding developmental period in the human was estimated (dark shading) based on the normalized thickness of the external granule layer (EGL) in the human and mouse. The results suggest a corresponding developmental window of vulnerability would extend from approximately 20 weeks gestation to 6.5 weeks after birth

Concluding remarks. In this paper, we describe the existence of a previously unrecognized window of vulnerability during which exposure to clinically relevant doses of GCs can produce rapid cerebellar brain degeneration within hours after exposure. Although more work needs to be done to confirm our cerebellar toxicity occurs during the human perinatal period, the striking similarities to the rodent cerebellar anatomy, function, and neurodevelopment ${ }^{26}$ suggest caution should be used when administering GCs in the perinatal clinical setting. It should be noted that previous research has found that prolonged increases in GC levels can lead to neurodegeneration in the hippocampus. But, whereas our NPC apoptosis occurs within hours, this hippocampal neurotoxicity occurs only after weeks to months of exposure. ${ }^{36}$ Finally, the results of this research may suggest endogenous GCs play an important neurodevelopmental role in cerebellar development by regulating NPC apoptosis. If true, this would be an important finding as there is a keen interest in the discovery of novel endogenous extracellular signaling mechanisms that regulate NPC apoptosis. ${ }^{23-25}$

\section{Materials and Methods}

Animals and drugs. C57BL/6 mice (Harlan, IN, USA) at PND7 were used in all experiments unless otherwise indicated. All animal care procedures were in accordance with standards approved by the Washington University Animal Studies Committee or the University of Alabama at Birmingham Institutional Animal Care and Use Committee. Generation of Bax/Bak ${ }^{37}$ and Puma ${ }^{20}$ knockout (KO) mice have been described previously. p53 KO mice were purchased from Taconic (Germantown, NY, USA).

Dexamethasone was administered intraperitoneally using the preservative-free prodrug dexamethasone sodium phosphate USP (Voigt Global Distribution LLC, Lawrence, KS, USA), the water soluble inorganic ester of DEX typically used clinically. All dexamethasone sodium phosphate concentrations are expressed as molar equivalents to DEX. Corticosterone was obtained from Sigma-Aldrich (St. Louis, MO, USA). The vehicles for DEX and corticosterone were saline USP and sesame oil, respectively. All drugs were administered at $10 \mu$ of vehicle per gram weight of animal. Unless otherwise indicated, following all drug treatments, animals were maintained for $2-12 \mathrm{~h}$ in a separate cage from the mother at an ambient temperature of $30^{\circ} \mathrm{C}$ until perfusion.

Immunohistochemistry. For all immmunohistochemistry, animals were deeply anesthetized, transcardially perfused with $4 \%$ paraformaldehyde in $0.1 \mathrm{M}$ Tris buffer, and the brains removed for postfixation and sectioning at $75 \mu \mathrm{m}$ in the sagittal plane using a vibratome. Sections were quenched using a methanol solution containing $3 \%$ hydrogen peroxide for $15 \mathrm{~min}$, placed in a blocking solution ( $2 \% \mathrm{BSA}$ $0.2 \%$ milk, $0.1 \%$ Triton X-100 in PBS) for an hour, and incubated in primary antibody overnight. The next morning, sections were placed in secondary antibody and reacted with $A B C$ reagents (standard Vectastain $A B C$ Elite Kit, Vector Laboratories, 
Burlingame, CA, USA) for an hour in each solution. Finally, the sections were incubated in VIP reagent (Vector VIP substrate kit for peroxidase, Vecto Laboratories) to visualize immunoreactivity. For immunofluorescence, sections were placed in blocking solution for an hour, placed in monoclonal/polyclonal primary antibodies overnight, and incubated for an hour in secondary antibodies for fluorescent tagging. Photocomposites were combined in Photoshop primarily using the Photomerge function.

Antibodies. Activated caspase-3 (Cell Signaling Technology Inc., Danvers, MA, USA; 1 : 1000). Calbindin (Sigma-Aldrich; $1: 1000)$. NeuN (Chemicon, Billerica, MA, USA; $1: 100$ ). Glial fibrillary acidic protein (Sigma-Aldrich; $1: 400)$. GC receptor (Santa Cruz Biotechnology, Santa Cruz, CA, USA; 1 : 8000). Ki-67 (BD Biosciences, San Jose, CA, USA; $1: 800$ ). p53 (Novocastra Laboratories, Newcastle upon Tyne, UK; $1: 1000$ ). Goat anti-rabbit biotinylated secondary antibody (Vector Laboratories; $1: 200$ ). Goat anti-mouse biotinylated secondary antibody (Vector Laboratories; $1: 200$ ). Goat anti-rabbit alexa fluor 488 secondary antibody (Molecular Probes, Carlsbad, CA, USA; $1: 750)$. Goat anti-mouse alexa fluor 594 secondary antibody (Molecular Probes; 1:750). Vectashield Mounting Medium with DAPI (Vector Laboratories).

Electron microscopy/semithin sections. For all electron microscopy and semithin sections, animals were deeply anesthetized and perfused with a fixative containing $1.5 \%$ glutaraldehyde and $1 \%$ paraformaldehyde. The brains were coronally cut into $1 \mathrm{~mm}$ slabs, osmicated overnight in $1 \%$ osmium tetroxide, dehydrated in graded ethanols, cleared in toluene, and embedded in araldite. Semithin sections (1- $\mu \mathrm{m}$ thick) were cut using glass knives on a MT-2B Sorval ultratome, heat dried, and stained with methylene blue/Azure B. For electron microscopy, ultrathin sections were cut with a diamond knife on a Leica Ultracut UCT ultramicrotome, suspended over a formvar-coated slot grid, stained with uranyl acetate and lead citrate, and visualized using a JEOL $100 \mathrm{CX}$ transmission electron microscope (Peabody, MA, USA).

\section{Semiquantitative evaluation of EGL degeneration} scores. Semiquantitative degeneration scores were established to produce a dose-response relationship and to evaluate a developmental window of vulnerability. An EGL degeneration score was evaluated for each animal by examining cerebellar sections from several different sagittal levels. A single rater blind to treatment, examined all regions of the EGL for $\mathrm{C} 3 \mathrm{~A}$ immunoreactive profiles, and semiquantitatively evaluated the extent of degeneration based on the following scale. Scales 0 , no apoptotic profiles are seen in all EGL regions; 1, apoptotic profiles are only seen in a minority of EGL regions; 2 , apoptotic profiles are seen in a majority of EGL regions; 3 , apoptotic profiles are seen in all regions of the EGL.

Estimation of an equivalent human developmental window of vulnerability. On the basis of our mouse developmental window of vulnerability, an equivalent period was extrapolated for the human based on normalized EGL thickness ratios from both species. To achieve this, the thickness of the EGL at different developmental periods was plotted as a normalized ratio to maximal thickness based on previously published research in the human ${ }^{38}$ and mouse. ${ }^{39}$ To graph this data, it was assumed that a human full-term birth occurs at 37 weeks of pregnancy and the period of synaptogenesis ends at 2 weeks or 2 years after birth in the mouse or human, respectively. Finally, based on our rodent window of vulnerability, an equivalent period in the human was calculated based on equal normalized EGL thickness ratios between species.

Stereology. To assess cerebellar neuronal counts, two series of cerebellar sections were chosen in a systematic random fashion from each brain. One series was immunohistochemically labeled for NeuN whereas the other was labeled for calbindin. The intersection interval, counting frame size, and distance between counting frames was adjusted so that a reasonable number (approximately 200) of degenerating cells were sampled. The optical fractionator method was used to provide an unbiased estimate of the total number of NeuN immunoreactive internal granule cells and calbindin immunoreactive Purkinje cells. Stereologic counting and estimates were done with the aid of Stereoinvestigator version 7.5 (MicroBrightField Inc., Colchester, VT, USA). A one-way repeated measures ANOVA was used for analyzing cell counts for each layer using the cell count as the dependent variable, drug treatment as the independent factor, and litter as a repeated measure. Significant F-ratios were followed by a Bonferroni's post hoc comparison to determine significant differences between drug treatments.
Behavioral locomotor activity and exploration test. Mice were evaluated during the early postweaning period (PND24) over a 1-h interval in transparent $(47.6 \times 25.4 \times 20.6 \mathrm{~cm}$ high) polystyrene enclosures using previously published methods. ${ }^{40}$ Each enclosure was surrounded by a frame containing a $4 \times 8$ matrix of photocell pairs, the output of which was fed to an online computer (Hamilton-Kinder, LLC, Poway, CA, USA). The system software defined a $33 \times 11 \mathrm{~cm}$ central zone, and a peripheral zone $(5.5 \mathrm{~cm}$ wide) surrounding the entire perimeter of the cage with the sides of the cage being the outermost boundary. Variables that were analyzed included the total number of ambulations, as well as the number of entries, time spent, and distance traveled in the center area, and the distance traveled in the periphery. Rearing was quantified using another frame containing pairs of photocells that was raised $8.2 \mathrm{~cm}$ above the floor of the enclosure.

Intracellular signaling mechanisms. Equal amounts $(25 \mu \mathrm{g})$ of cytosolic lysates were resolved by SDS-PAGE and transferred to PVDF. Blots were blocked in $5 \%$ milk in wash buffer (200 mM Tris Base, $1.37 \mathrm{M} \mathrm{NaCl}, 1 \%$ Tween 20, pH7.6) for $1 \mathrm{~h}$, followed by overnight incubation with primary antibodies (C3A, Cell Signaling Technology Inc. and $\beta$-tubulin, Santa Cruz Biotechnology). After incubation, blots were washed with wash buffer, and then incubated with horseradish peroxidase conjugate secondary antibody (goat anti-rabbit immunoglobulin G, Bio-Rad Laboratories, Hercules, CA, USA) for $1 \mathrm{~h}$ at room temperature. Cleaved caspase3 was detected using Supersignal chemiluminescence (Pierce Biotechnology, Rockford, IL, USA) and $\beta$-tubulin was detected by ECL (Amersham, Piscataway, $\mathrm{NJ}$, USA). Western blots were scanned into Adobe Photoshop and digitized with UN-SCAN-IT software, version 6.1 (Silk Scientific, Orem, UT, UDA). For western blot experiments, involving quantification, the mean \pm S.D. was determined from at least three independent experiments, with an ' $n$ ' of one representing one WT saline treated, WT Dex treated, or KO Dex treated. Effects of genotype and treatment were analyzed for significance using two-way ANOVA, followed by Bonferroni's test for pair-wise comparisons. In all cases, a $P$-value of less than or equal to 0.05 was considered significant.

Acknowledgements. KCW and KAR thank UAB Neuroscience Core Facilities (NS47466 and NS57098) for technical support. KAR and KCW are supported by $\mathrm{NIH}$ grants NS35107 and NS41962. Puma - / - mice were generously provided to us by Dr. Andreas Strasser (University of Melbourne). KKN, NBF, and JWO thank D Smith and $\mathrm{H}$ Wang for technical assistance. KKN is supported by NIH grants DA07261, DA05072, and HD055365. DFW is supported by an NIH P30 Neuroscience Blueprint Core Grant NS057105. NBF is supported by NIH grant ES12443 and HD055365. JWO is supported by NIH grant DA05072 and HD055365. Finally, we thank K Dikranian and A Parsadanian for advice and technical support with double immunofluorescent histology.

1. Effect of corticosteroids for fetal maturation on perinatal outcomes. NIH Consens State 1994; 12: 1-24.

2. Antenatal corticosteroids revisited: repeat courses. NIH Consens State 2000; 17: 1-18

3. Leviton LC, Goldenberg RL, Baker CS, Schwartz RM, Freda MC, Fish LJ et al. Methods to encourage the use of antenatal corticosteroid therapy for fetal maturation: a randomized controlled trial. JAMA 1999; 281: 46-52.

4. Halliday $\mathrm{HL}$. The effect of postnatal steroids on growth and development. J Perinat Med 2001; 29: 281-285

5. Grier DG, Halliday HL. Corticosteroids in the prevention and management of bronchopulmonary dysplasia. Semin Neonatol 2003; 8: 83-91.

6. Halliday HL. Use of steroids in the perinatal period. Paediatr Respir Rev 2004; 5 (Suppl A): S321-S327.

7. Postnatal corticosteroids to treat or prevent chronic lung disease in preterm infants. Pediatrics 2002; 109: 330-338.

8. Yeh TF, Lin YJ, Lin HC, Huang CC, Hsieh WS, Lin CH et al. Outcomes at school age after postnatal dexamethasone therapy for lung disease of prematurity. N Engl J Med 2004; 350: 1304-1313.

9. St John EB, Carlo WA. Respiratory distress syndrome in VLBW infants: changes in management and outcomes observed by the NICHD Neonatal Research Network. Semin Perinatol 2003; 27: 288-292.

10. Jobe AH, Soll RF. Choice and dose of corticosteroid for antenatal treatments. Am J Obstet Gynecol 2004; 190: 878-881.

11. Ikonomidou C, Bittigau P, Ishimaru MJ, Wozniak DF, Koch C, Genz K et al. Ethanolinduced apoptotic neurodegeneration and fetal alcohol syndrome. Science 2000; 287: 1056-1060. 
12. Ikonomidou C, Bosch F, Miksa M, Bittigau P, Vockler J, Dikranian K et al. Blockade of NMDA receptors and apoptotic neurodegeneration in the developing brain. Science 1999 283: $70-74$.

13. Jevtovic-Todorovic V, Hartman RE, Izumi Y, Benshoff ND, Dikranian K, Zorumski CF et al. Early exposure to common anesthetic agents causes widespread neurodegeneration in the developing rat brain and persistent learning deficits. J Neurosci 2003; 23: 876-882.

14. Olney JW, Tenkova T, Dikranian K, Muglia LJ, Jermakowicz WJ, D'Sa C et al. Ethanolinduced caspase-3 activation in the in vivo developing mouse brain. Neurobiol Dis 2002; 9: 205-219.

15. De Kloet ER, Vreugdenhil E, Oitzl MS, Joels M. Brain corticosteroid receptor balance in health and disease. Endocr Rev 1998; 19: 269-301.

16. Pavlik A, Buresova M. The neonatal cerebellum: the highest level of glucocorticoid receptors in the brain. Brain Res 1984; 314: 13-20.

17. Goldowitz D, Hamre K. The cells and molecules that make a cerebellum. Trends Neurosci 1998; 21: 375-382

18. Shacka JJ, Roth KA. Bcl-2 family and the central nervous system: from rheostat to rea complex. Cell Death Differ 2006; 13: 1299-1304.

19. Akhtar RS, Ness JM, Roth KA. Bcl-2 family regulation of neuronal development and neurodegeneration. Biochim Biophys Acta 2004; 1644: 189-203.

20. Villunger A, Michalak EM, Coultas L, Mullauer F, Bock G, Ausserlechner MJ et al. p53- and drug-induced apoptotic responses mediated by $\mathrm{BH} 3-$ only proteins puma and noxa. Science 2003; 302: 1036-1038.

21. Geng Y, Akhtar RS, Shacka JJ, Klocke BJ, Zhang J, Chen X et al. p53 transcriptiondependent and -independent regulation of cerebellar neural precursor cell apoptosis J Neuropathol Exp Neurol 2007; 66: 66-74.

22. Andersen BB, Korbo L, Pakkenberg B. A quantitative study of the human cerebellum with unbiased stereological techniques. J Comp Neurol 1992; 326: 549-560.

23. de la Rosa EJ, de Pablo F. Cell death in early neural development: beyond the neurotrophic theory. Trends Neurosci 2000; 23: 454-458.

24. De Zio D, Giunta L, Corvaro M, Ferraro E, Cecconi F. Expanding roles of programmed cell death in mammalian neurodevelopment. Semin Cell Dev Biol 2005; 16: 281-294.

25. Depaepe V, Suarez-Gonzalez N, Dufour A, Passante L, Gorski JA, Jones KR et al. Ephrin signalling controls brain size by regulating apoptosis of neural progenitors. Nature 2005; 435: $1244-1250$.

26. Chizhikov V, Millen KJ. Development and malformations of the cerebellum in mice. Mol Genet Metab 2003; 80: 54-65.
27. Benesova O, Pavlik A Perinatal treatment with glucocorticoids and the risk of maldevelopment of the brain. Neuropharmacology 1989; 28: 89-97.

28. Schmahmann JD. Disorders of the cerebellum: ataxia, dysmetria of thought, and the cerebellar cognitive affective syndrome. J Neuropsychiatry Clin Neurosci 2004; 16: 367-378.

29. Hoyert DL, Mathews TJ, Menacker F, Strobino DM, Guyer B. Annual summary of vital statistics: 2004. Pediatrics 2006; 117: 168-183.

30. Schmidt MV, Oitzl MS, Levine S, de Kloet ER. The HPA system during the postnatal development of CD1 mice and the effects of maternal deprivation. Brain Res 2002; 139: 39-49.

31. Robson AC, Leckie CM, Seckl JR, Holmes MC. 11 beta-hydroxysteroid dehydrogenase type 2 in the postnatal and adult rat brain. Brain Res 1998; 61: 1-10.

32. Holmes MC, Sangra M, French KL, Whittle IR, Paterson J, Mullins JJ et al. 11 betaHydroxysteroid dehydrogenase type 2 protects the neonatal cerebellum from deleterious effects of glucocorticoids. Neuroscience 2006; 137: 865-873.

33. Medh RD, Thompson EB. Hormonal regulation of physiological cell turnover and apoptosis Cell Tissue Res 2000; 301: 101-124.

34. Liggins GC. The role of cortisol in preparing the fetus for birth. Reprod Fertil Dev 1994; 6 141-150.

35. Erlacher M, Labi V, Manzl C, Bock G, Tzankov A, Hacker G et al. Puma cooperates with $\mathrm{Bim}$, the rate-limiting $\mathrm{BH} 3$-only protein in cell death during lymphocyte development, in apoptosis induction. J Exp Med 2006; 203: 2939-2951.

36. Sapolsky RM. Glucocorticoids, stress, and their adverse neurological effects: relevance to aging. Exp Gerontol 1999; 34: 721-732

37. Akhtar RS, Geng Y, Klocke BJ, Roth KA. Neural precursor cells possess multiple p53-dependent apoptotic pathways. Cell Death Differ 2006; 13 1727-1739.

38. Rakic $P$, Sidman RL. Histogenesis of cortical layers in human cerebellum, particularly the lamina dissecans. J Comp Neurol 1970; 139: 473-500.

39. Fujita S, Shimada M, Nakamura T. H3-thymidine autoradiographic studies on the cell proliferation and differentiation in the external and the internal granular layers of the mouse cerebellum. J Comp Neurol 1966; 128: 191-208.

40. Wozniak DF, Hartman RE, Boyle MP, Vogt SK, Brooks AR, Tenkova T et al. Apoptotic neurodegeneration induced by ethanol in neonatal mice is associated with profound learning/memory deficits in juveniles followed by progressive functional recovery in adults. Neurobiol Dis 2004 : 17 : 403-414. 